Service social

\title{
Inégalités de santé et parcours de vie : réflexions sur quelques déterminants sociaux de l'expérience d'hommes considérés comme vulnérables
}

\author{
Bernard Roy, Maria De Koninck, Michelle Clément et Éric Couto
}

Volume 58, numéro 1, 2012

URI : https://id.erudit.org/iderudit/1010438ar

DOI : https://doi.org/10.7202/1010438ar

Aller au sommaire du numéro

Éditeur(s)

École de service social de l’Université Laval

ISSN

1708-1734 (numérique)

Découvrir la revue

Citer cet article

Roy, B., De Koninck, M., Clément, M. \& Couto, É. (2012). Inégalités de santé et parcours de vie : réflexions sur quelques déterminants sociaux de l'expérience d'hommes considérés comme vulnérables. Service social, 58(1), 32-54.

https://doi.org/10.7202/1010438ar
Résumé de l'article

Dans le cadre d'une recherche portant sur les inégalités de santé en milieu de vie de trois territoires de la région de Québec, la présence d'écarts appréciables est ressortie au sein de deux sous-populations : les familles monoparentales ayant de jeunes enfants et les hommes de 45 à 64 ans d'un quartier urbain. Devant ces résultats, l'équipe de recherche a élaboré une seconde étude afin de repérer les facteurs structurels et sociaux pouvant être en jeu dans la production de ces inégalités. Ce sont les résultats portant sur les hommes et découlant de cette seconde recherche qui sont discutés dans le présent article. Une approche par parcours de vie a été adoptée et les données recueillies ont été analysées chronologiquement et selon différents parcours de vie (famille d’origine, vécu scolaire, travail, parcours résidentiel). Les caractéristiques personnelles, les liens sociaux et les ressources sociosanitaires utilisées par les répondants ont également été pris en compte. Chacun des parcours présentés par chaque entrevue a aussi été caractérisé par la résilience ou la non-résilience selon une démarche consensuelle interjuge afin d'en dégager des facteurs de vulnérabilité ou de protection. L'article se conclut par une discussion établissant des liens entre les résultats obtenus et d'autres recherches qui se sont intéressées aux réalités vécues par les hommes. 


\title{
Inégalités de santé et parcours de vie : réflexions sur quelques déterminants sociaux de l'expérience d'hommes considérés comme vulnérables*
}

\author{
Bernard Roy \\ Maria De Koninck \\ Michelle Clément \\ Éric Couto
}

\section{RÉSUMÉ}

Dans le cadre d'une recherche portant sur les inégalités de santé en milieu de vie de trois territoires de la région de Québec, la présence d'écarts appréciables est ressortie au sein de deux sous-populations : les familles monoparentales ayant de jeunes enfants et les hommes de 45 à 64 ans d'un quartier urbain. Devant ces résultats, l'équipe de recherche a élaboré une seconde étude afin de repérer les facteurs structurels et sociaux pouvant être en jeu dans la production de ces inégalités. Ce sont les résultats portant sur les hommes et découlant de cette seconde recherche qui sont discutés dans le présent article. Une approche par parcours de vie a été adoptée et les données recueillies ont été analysées chronologiquement et selon différents parcours de vie (famille d'origine, vécu scolaire, travail, parcours résidentiel). Les caractéristiques personnelles, les liens sociaux et les ressources sociosanitaires utilisées par les répondants ont également été pris en compte. Chacun des parcours présentés par chaque entrevue a aussi été caractérisé par la résilience ou la non-résilience selon une démarche consensuelle interjuge afin d'en dégager des facteurs de vulnérabilité ou de protection. L'article se conclut par une discussion établissant des liens entre les résultats obtenus et d'autres recherches qui se sont intéressées aux réalités vécues par les hommes.

Mots-clés : santé des hommes; inégalités de santé ; parcours de vie ; déterminants de la santé ; résilience

\section{INTRODUCTION}

Au Québec, peu d'études ont porté spécifiquement sur la question de la santé des hommes. Ce n'est qu'en 2001 que le ministère de la Santé et des Services sociaux s'est senti concerné par l'état de santé et de bien-être des hommes; il a alors mis sur pied le Comité de travail en matière de prévention et d'aide aux hommes (Lefebvre, 2004). Une espérance de vie plus courte et une surmortalité (en particulier par suicide, mais également pour cause d'accidents ou de diverses maladies) constituent les deux éléments clés justifiant la prise en compte de la santé des hommes dans ce qu'elle présente de spécifique.

Dans le cadre d'un projet intitulé «Inégalités de santé et milieux de vie: déterminants sociaux en cause et leurs interactions », réalisé de 2002 à 2006 dans trois territoires de la

\footnotetext{
La recherche sur laquelle s'appuyaient les résultats présentés avait reçu un soutien financier du Fonds québécois de recherche sur la société et la culture (FQRSC).
} 
région de Québec, soit un quartier situé au centre-ville (Saint-Louis), un autre en banlieue (Banville) et un dernier en milieu rural (Verdier) ${ }^{1}$ (De Koninck, 2008), la présence d'écarts de santé appréciables est ressortie entre ceux-ci et au sein de chacun. Notamment, deux souspopulations sont apparues particulièrement défavorisées sur le plan de la santé : les familles monoparentales ayant de jeunes enfants et résidant dans les trois territoires et les hommes de 45 à 64 ans du quartier Saint-Louis. En ce qui concerne ces derniers, les chercheurs furent intéressés par certains résultats obtenus lors de l'enquête téléphonique de l'étude. En effet, plus de $90 \%$ des individus âgés de 44 ans et moins qualifiaient leur état de santé général de « bon à excellent », alors qu'après 45 ans, cette proportion était de $72 \%$. D'autres indicateurs de santé défavorables ont également été observés dans ce groupe. L'espérance de vie des hommes à la naissance y était, en moyenne, de 3,9 ans inférieure à celle des résidants de Banville. Les données sur la mortalité, l'incapacité à long terme et l'hospitalisation révélaient aussi que la santé des résidants de Saint-Louis était prématurément affectée. On y observait une forte incidence de cancer ainsi qu'un taux exceptionnellement élevé d'hospitalisation pour troubles mentaux (De Koninck, 2008).

Comment pouvait-on expliquer de tels écarts de santé pour cette population? Une certitude se dégageait: "La construction des inégalités est le fruit de la conjugaison horizontale et verticale d'un ensemble de facteurs. Ne s'intéresser qu'à l'un de ceux-ci est vain. Ces facteurs sont structurels et sociaux, les deux types étant en interaction » (De Koninck, 2008 : 77). II est donc apparu évident que seule une démarche produisant des données complexes pouvait contribuer au repérage et à la compréhension des facteurs structurels et sociaux en jeu dans la production de ces inégalités. Conséquemment, un nouveau projet fut élaboré. Reposant également sur une enquête téléphonique et une démarche qualitative avec entrevues individuelles, il avait pour but de mieux saisir et comprendre les facteurs sociaux et dynamiques en cause dans ces situations de défavorisation ${ }^{2}$ plus marquées chez les hommes de 45 à 64 ans du quartier Saint-Louis et les familles monoparentales des trois territoires.

Ce sont les résultats portant sur les hommes et découlant du volet qualitatif de cette seconde recherche qui sont discutés dans le présent article. Dans un premier temps, nous présenterons le devis de cette étude (objectifs, cadre conceptuel, concepts, méthodologie). Puis, nous exposerons certains résultats qui concernent différents aspects de l'expérience des sujets rencontrés. Nous terminerons cet article par une discussion établissant des liens entre les résultats obtenus et d'autres recherches qui se sont intéressées aux réalités vécues par les hommes.

1. Les noms de quartiers sont fictifs afin de sauvegarder la confidentialité des participants et de ne pas contribuer à la stigmatisation de ces milieux de vie. Ces trois territoires furent sélectionnés à l'aide d'indices de défavorisation sociale et matérielle élaborés par Pampalon et Raymond (2000).

2. Les indices de défavorisation matérielle et sociale ont été développés par Pampalon et Raymond (2001) à des fins d'analyse des inégalités et d'intervention socio-sanitaire (voir http://www.inspq.qc.ca/pdf/publications/295IndiceDefavorisation_2001.pdf) ou (http://www.msss.gouv.qc.ca/statistiques/atlas/atlas/). Ils permettent de mesurer les disparités sociales associées à la mortalité, à la morbidité sociale et sanitaire et au recours aux services de santé et de services sociaux à court et à long terme. 


\section{Présentation de la Recherche}

\section{Objectifs de l'étude}

Selon la littérature portant sur les inégalités sociales de santé et les milieux de vie, la présence de populations en situation de pauvreté et d'exclusion sociale agit dans un milieu par l'intermédiaire de dynamiques sociales (représentations, perceptions de problèmes, orientation des dynamiques collectives, etc.) et environnementales. Si la situation des populations défavorisées d'un milieu n'est pas améliorée, c'est l'ensemble du milieu qui peut en souffrir. Mais l'action ne peut pas se limiter au niveau local puisque des conditions structurelles contribuent aussi aux conditions de vie des populations (éducation, emploi, protections en cas de maladie, etc.).

L'étude dont il est question ici visait à améliorer les connaissances sur la genèse et la réduction de la pauvreté et de l'exclusion sociale en s'intéressant aux deux sous-populations susmentionnées et identifiées comme défavorisées. Plus spécifiquement, elle devait documenter les facteurs de risque et de protection présents dans les milieux locaux ciblés, tout en prenant en compte les facteurs de nature plus globale. Concrètement, elle cherchait à répondre aux questions suivantes: 1) Au-delà des facteurs identifiés dans la première recherche (conditions matérielles, liens sociaux, perceptions de cohésion sociale, problèmes sociaux et environnementaux) (Équipe de recherche sur les inégalités sociales de santé, 2006a, 2006b, 2006c, 2006d), quels sont les facteurs sociaux et les dynamiques en cause dans la genèse de la défavorisation plus marquée des deux sous-groupes retenus (familles monoparentales et hommes de 45 à 64 ans)? 2) Au sein de ces deux sous-groupes, en quoi se distinguent les parcours de vie des personnes qui ne se considèrent ni pauvres ni exclues?

\section{Cadre conceptuel}

Pour répondre aux objectifs de la démarche qualitative de cette recherche, la perspective de parcours de vie (Bernard et al., 2006) a été adoptée. Celle-ci permet d'intégrer les facteurs de risque sociaux et biologiques plutôt que d'établir de fausses dichotomies entre eux (Kuh, BenShlomo, Lynch, Hallqvist et Power, 2003). Quatre principes sous-tendent cette perspective : 1) la vie se déroule dans le temps; 2) la vie est faite de multiples aspects intégrés; 3) les trajectoires de vie sont interreliées et 4) les trajectoires de vie s'inscrivent dans des milieux socialement façonnés (Bernard et al., 2006). De plus, cette approche stipule que la santé est le résultat des conditions actuelles de vie et celui des conditions passées (Krieger, 2001). L'approche par parcours de vie offre donc un moyen de conceptualiser la façon dont les déterminants de la santé biologiques et socio-environnementaux, vécus à différentes étapes de la vie, peuvent influencer de façon différentielle le développement de maladies, de vulnérabilités ou d'exclusion sociale. «[Un] des atouts de cette approche est qu'elle montre également comment les risques qui surviennent tout au long de la vie peuvent être déterminés, corrigés ou modifiés au cours de la période nécessaire au développement d'une maladie chronique » (Reading, 2009 : A-61). Enfin, une approche par parcours de vie peut contribuer à déterminer 
quelles sont les politiques et méthodes de prévention les plus efficaces pour combattre l'avènement des maladies chroniques.

Le cadre retenu présente donc l'état de santé physique et mental des individus appartenant à des sous-groupes de populations comme découlant de leurs parcours de vie (familial,

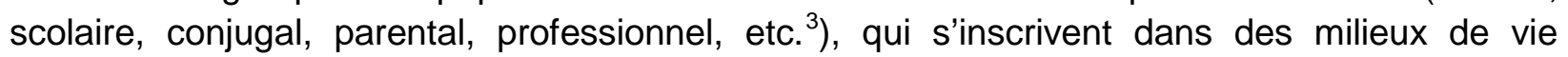
(parcours résidentiel) et au cours desquels ils ont été en interaction avec des ressources (sociales, sanitaires). Par conséquent, l'analyse des parcours de vie a été réalisée afin de mettre en relief les facteurs responsables de la situation de défavorisation des hommes.

\section{Pauvreté et vulnérabilité}

Deux concepts méritent d'être présentés afin de qualifier ce que nous entendons par défavorisation en tant qu'expérience vécue, soit ceux de pauvreté et de vulnérabilité. La pauvreté peut être définie comme un état caractérisé par la privation individuelle et sociale mettant en cause les possibilités de combler ses besoins (physiques, psychologiques et sociaux) et de développer les capacités permettant de mettre en œuvre ses aspirations (Dupéré et Disant, 2005). Cette privation crée une fragilité et, conséquemment, une vulnérabilité à l'exclusion sociale. Étant donné le constat d'états de santé différenciés attribuables, du moins en partie, à des inégalités sociales, la pauvreté et l'exclusion sociale sont ici compris en tant qu'états de privation ayant des répercussions sur les capacités et les fonctionnements essentiels à l'acquisition et au maintien d'un bon état de santé.

Concernant le concept de vulnérabilité, Roy écrit ceci :

Une sorte de consensus [...] se dégage sur l'importance de l'individualisme comme fondement des rapports sociaux contemporains et de l'injonction individuelle à se réaliser, être autonome et responsable de son succès ou de son échec. Cela constituerait la base de la vulnérabilité. [...] elle serait synonyme d'instabilité, d'insuffisance ou d'assouplissement des supports sociaux qui permettent à l'individu de se réaliser. La décollectivisation et l'individualisation des rapports sociaux créeraient de nouveaux contextes dans lesquels chacun doit s'inscrire. Certains individus y arriveraient facilement, mais d'autres, ne disposant pas globalement ou temporellement des ressources nécessaires, seraient en situation vulnérable ou de vulnérabilité. (2008: 24)

Ainsi, être ou devenir vulnérable exposerait les individus à la pauvreté et à l'exclusion sociale (dynamique à deux sens). C'est pourquoi, connaître les conditions qui fragilisent les individus (et éventuellement leurs communautés) devient particulièrement intéressant. Ce sont les processus structurels, sociaux et individuels qui participent à des épisodes plus ou moins longs de fragilité qui ont fait l'objet des questionnements de la présente étude.

3. Plus précisément, le présent article traitera de leur parcours familial et scolaire, de leur parcours travail, de leur parcours résidentiel ainsi que de leur santé physique et mentale, des ressources qu'ils ont utilisées ainsi que des liens sociaux tissés au sein de leur communauté. D'autres aspects du vécu des hommes rencontrés (parcours conjugal et de paternité) feront l'objet de publications ultérieures. 


\section{Résilience}

Considérant que des conditions matérielles difficiles, le cumul de difficultés et la faiblesse ou l'absence de liens sociaux protecteurs poussent ou maintiennent certaines populations dans une situation délétère, le concept de résilience a été mis à contribution pour distinguer les parcours de vie des personnes ne se considérant ni pauvres ni exclues de celles qui ont une perception négative de leurs conditions. Les études qui utilisent ce concept le font généralement dans une démarche de recherche de facteurs protecteurs pouvant modifier les effets négatifs d'événements de la vie (Luthar, 2006). Cela ne fait toutefois pas consensus. Rutter (1987, 1990, 2000), par exemple, caractérise la résilience comme l'aboutissement positif du processus développemental d'individus considérés comme à haut risque. Quant à eux, Masten et ses collaborateurs (Masten, 1994; Masten, Best et Garmezy, 1990) distinguent trois groupes de phénomènes de résilience : 1) les situations où des personnes à risque présentent des résultats supérieurs à ceux anticipés; 2) le maintien d'une adaptation positive à un environnement hostile, et 3) le fait pour des personnes de retomber sur leurs pieds à la suite d'un traumatisme. Finalement, pour Cyrulnik, la résilience est définie comme « [...] la capacité d'affronter avec succès les risques et les déboires sérieux de l'existence. II s'agit d'une combinaison de force intérieure, d'appui de l'extérieur et d'apprentissage à partir de l'expérience acquise » (1999: 23).

Nous avons appréhendé la résilience non pas comme un état ou un trait de personnalité mais plutôt comme un processus permettant aux individus de traverser des événements difficiles et impliquant trois éléments : 1) les événements de la vie; 2) les facteurs personnels et 3) les liens à l'environnement. Le concept de résilience nous est également apparu intéressant en ce sens qu'il oblige à se centrer sur les ressources mobilisables, les compétences et les forces des populations.

\section{Méthodologie}

Les données proviennent d'entrevues individuelles en face à face auprès d'hommes de 45 à 64 ans résidant à Saint-Louis, recrutés parmi un groupe de 101 hommes ayant été contactés dans le cadre d'un volet précédent de l'étude ${ }^{4}$. Pour participer à l'étude, les hommes devaient habiter depuis au moins trois ans dans le quartier Saint-Louis puisque nous nous intéressions à la pauvreté en lien avec le milieu de vie. Les critères de l'occupation, de la scolarité et de l'âge ont aussi été considérés afin de s'assurer de rencontrer des hommes vivant différentes situations.

Compte tenu de notre objectif de déceler en quoi se distinguent les parcours de vie des personnes qui ne se considèrent ni pauvres ni exclues, quatre catégories de répondants ont été créées selon les réponses données lors de l'enquête : 1) Avoir manqué d'argent et se percevoir comme pauvre ou très pauvre; 2) Avoir manqué d'argent et se percevoir comme étant à l'aise financièrement ou comme ayant des revenus suffisants pour répondre à ses besoins; 3) Ne pas avoir manqué d'argent et se percevoir comme étant à l'aise financièrement ou comme ayant des revenus suffisants pour répondre à ses besoins et 4) Ne pas avoir manqué d'argent et se percevoir comme pauvre ou très pauvre. Dix hommes sur les 19 se classant dans les trois

4. L'enquête téléphonique. 
premières catégories ont accepté de nous rencontrer et 12 entrevues ont été réalisées avec des hommes de la catégorie 4. Cet échantillon a été complété par deux autres entrevues avec des sujets recrutés grâce au soutien d'organismes communautaires, pour un total de 24 entrevues.

Les entrevues ont duré entre $1 \mathrm{~h} 15$ et $4 \mathrm{~h}$ et ont eu lieu aux endroits choisis par les participants. Sauf dans deux cas, elles ont été réalisées par le même intervieweur. L'entrevue était amorcée par la question suivante :

Nous avons mené une recherche sur la santé dans Saint-Louis, nous avons trouvé toutes sortes de choses. Suite à cela, nous avons constaté que nous ne connaissions pas bien certains éléments, dont l'histoire de la vie des personnes qui y habitent. Nous avons besoin, entre autres, d'en savoir plus sur les hommes de 45 à 64 ans qui vivent ici et qu'ils nous racontent leur histoire. Pouvez-vous me raconter votre histoire?

Chaque entrevue a été analysée à l'aide d'une grille originale découpée de façon chronologique (par dizaines d'années de vie). Pour chacune, les propos ont été inscrits selon le parcours auquel ils faisaient référence. Les parcours retenus étaient les suivants: famille d'origine, parcours conjugal, parcours de paternité, parcours scolaire, parcours de travail et parcours résidentiel. Ont également été pris en compte les caractéristiques personnelles, les liens sociaux et les ressources utilisées par les répondants. L'analyse verticale de chaque entrevue a permis de faire ressortir les singularités dans les parcours. L'analyse horizontale de l'ensemble des entrevues a mis au jour quelques récurrences significatives. Le parcours présenté par chaque entrevue a finalement été caractérisé par la résilience ou la non-résilience à l'issue d'une démarche consensuelle des chercheur-e-s.

II va sans dire qu'une telle stratégie de recherche ne nous permet pas de généraliser statistiquement les résultats obtenus. Par contre, les expériences recueillies ainsi que les caractéristiques des groupes d'hommes distingués selon le caractère plus ou moins résilient de leur parcours fournissent des informations qui permettent une certaine généralisation théorique.

\section{RÉSULTATS OBTENUS}

\section{Parcours familial}

Le rôle de la famille ressort comme une dimension très importante dans la vie des hommes. Les événements qui se sont produits dans l'enfance, souvent liés à la famille d'origine, semblent avoir eu un impact déterminant sur les expériences suivantes. Certains nous ont décrit des milieux aimants ayant favorisé le développement d'habiletés qui leur permettraient, en tant qu'adultes, de se réaliser, de sortir de situations difficiles ou d'éviter certains risques. À contrario, d'autres ont grandi dans des familles dysfonctionnelles ou dans des conditions difficiles (difficultés économiques, maladie mentale, comportements abusifs, etc.). Trois facteurs touchant au rôle de la famille ressortent comme ayant une influence sur la construction de la vulnérabilité chez les hommes rencontrés ou leur capacité de résilience. 
Le premier est la force ou la faiblesse de l'ancrage familial, soit le sentiment d'appartenance à une famille et la possibilité de se tourner vers elle dans les moments significatifs de la vie. Le cas d'un participant orphelin est apparu particulièrement éloquent à ce propos. Ce dernier, placé toute son enfance en foyers d'accueil, a connu des sévices, psychologiques et sexuels. II raconte: "Après ça, y faut rester sain envers la société, faut rester sain envers la vie, et... Mais, quand tu portes des affaires de même... »(E-812) Privé de famille, il nous dira « appartenir à la société ». Cet homme profita d'une éclaircie dans sa vie lorsqu'une relation amoureuse lui permit de trouver un confort matériel et affectif inconnu jusque-là. La fin de cette relation, causée par le décès de son partenaire, le replongera toutefois dans une position de survie. D'une part, ce récit illustre bien les conditions favorables au développement optimal d'un enfant (sécurité affective, respect, etc.). D'autre part, il démontre que des conditions difficiles vécues dans l'enfance n'excluent pas tout accès à une vie pouvant assurer un certain bonheur. Dans ce cas-ci, cet accès ne s'est concrétisé que pour une certaine période et aurait peut-être pu durer si l'homme avait eu un meilleur capital social et culturel.

Un second facteur est la désorganisation dans certaines familles qui semble vouloir « piper » les dés. Un participant raconte :

Chez nous, pas de violence, mais y avait d'la boisson... la boisson, ça coulait à flots [...] C'est sûr quand on était jeunes on n'avait pas l'choix là, fallait vivre dedans. Mais aussitôt qu'on a pogné, j'sais pas moi, huit, neuf ans, 'garde, on... on n'était jamais chez nous. On v'nait juste pour manger. Pis on rentrait jamais nos amis, sauf une coup'e qui étaient habitués, ceux qui connaissaient la situation. À part ça, euh, tu rentrais personne. (E-341)

Des problèmes tels que la toxicomanie des parents, la violence entre les parents, envers les enfants, entre les enfants et la maladie mentale des parents constituent un lourd tribut qui marque ceux qui les ont vécus. Par exemple, un participant rapporte les embuches de son enfance : père violent, parents séparés alors qu'il avait deux ans, abus sexuel à l'âge de quatre ans par son frère de douze ans son aîné, mère hospitalisée en psychiatrie pour toxicomanie. Les propos recueillis laissent également penser que les hommes ayant vécu de telles difficultés sont préoccupés par leur impact potentiel sur leurs propres enfants.

Enfin, troisième facteur, l'affection reçue dans l'enfance participe grandement à la résilience des hommes rencontrés. Ainsi, un participant ayant connu une vie difficile et de nombreux revers économiques a profité du soutien de deux personnes significatives pour lui, soit une tante qui l'a accueilli au décès de sa mère alors qu'il n'avait que trois ans, ainsi que sa conjointe avec qui il partage sa vie et dont il est très proche. Ces liens semblent lui avoir permis de faire face à l'adversité et de toujours conserver cette vision positive qu'il a partagée avec nous :

Pis moi, j'ai une philosophie dans la vie, que tout s'arrange. $Y$ a toujours une solution à que'que chose. Des fois est ' t'être ben pas bonne, est 't'être ben... Non, j'dirais pas ça. $Y$ a toujours une solution dans notre vie, mais a fait peut-être ben pas toujours notre affaire. (E-18) 
Un autre cas témoigne du nécessaire équilibre dans l'éducation donnée aux enfants, alors qu'un homme nous a confié avoir été soumis à un encadrement excessif par un père catholique, adepte de la discipline et surprotecteur. Selon lui, cette façon d'agir l'a empêché d'acquérir son autonomie, ce qui fait en sorte que, bien qu'au début de la cinquantaine, il est encore soutenu financièrement par ce père, et qu'il n'a pas su développer de relation conjugale durable et stable et n'a pas eu d'enfant.

Il est également à noter que les pères de presque tous les sujets correspondent au modèle de l'homme pourvoyeur. Ce rôle, étant intimement associé à la masculinité dans les récits, a eu une incidence importante sur le parcours de vie de certains lorsqu'ils ont perdu ce statut ou se sont trouvés dans l'incapacité de l'assumer. II est aussi intéressant de voir que les mères sont beaucoup moins présentes que les pères dans les récits. Elles sont surtout décrites comme demeurant à la maison pour s'occuper des enfants et assumer le travail domestique. Bref, la mère est présentée comme la gardienne du lien familial. La maladie ou la mort de cette dernière est même associée à l'éclatement de la famille dans certains récits. Le rapport privilégié entre les mères et leurs filles a aussi été vécu difficilement par certains hommes.

Les entretiens nous révèlent que le parcours familial des hommes résilients est marqué, d'une part, par la stabilité du milieu, mais également par la présence d'une ou de plusieurs figures d'attachement. II arrive que certains problèmes soient présents dans leur milieu, surtout calqué sur un modèle traditionnel. Cependant, dans de tels cas, les propos à l'égard des parents ne sont pas négatifs et un désir de rapprochement ou de réconciliation est souvent exprimé. Dans ces cas, la famille élargie offre également du soutien. Règle générale, les hommes résilients ont un attachement profond à leur milieu d'origine et expriment la possibilité d'y être accueillis et soutenus. En ce qui concerne les non-résilients, leurs trajectoires familiales se situent dans un univers dysfonctionnel ou difficile. L'ambiance y est marquée par des disputes, de la violence, de la maladie mentale, la séparation des parents, l'absence, le départ ou le décès du père, etc. D'importantes blessures peuvent aussi être associées à la perte ou l'absence d'une figure d'attachement pour ces hommes.

\section{Parcours scolaire}

Les parcours scolaires des participants sont variés: l'un était analphabète (condition déterminante dans sa trajectoire); neuf participants n'avaient pas obtenu de diplôme d'études secondaires; cinq avaient complété cette étape; deux possédaient un diplôme d'études collégiales et sept avaient fait des études universitaires (l'un d'eux avait entrepris un $3^{\mathrm{e}}$ cycle sans le terminer).

Les discours sur ce parcours sont, en général, peu développés. Neuf participants mentionnent ne pas avoir aimé l'école. Pour plusieurs, il s'agit de périodes d'échecs, même s'ils ont obtenu un diplôme. Il en va de même pour ceux qui n'ont pas complété le cycle d'études amorcé ou qui ont voulu retourner aux études. Certains y sont arrivés, mais sans que cela leur permette d'améliorer leur sort. Fait notable, sauf exception, l'école ne semblait pas être une priorité pour les parents des participants. 
Le parcours scolaire des hommes résilients est plutôt sans problème. Bien qu'il ne soit pas forcément marqué de grands succès, les résilients auront parfois construit leur projet de vie autour des études et en auront gardé de bons souvenirs.

À contrario, aucun des non-résilients n'a de souvenir heureux associé à l'école. Ils y ont vécu violence, humiliation, de nombreux échecs, des expériences difficiles ou troublantes. Bien que peu loquaces sur cette question, certains ont su nous faire comprendre l'impact de ces expériences sur l'atténuation de leur résilience. À titre d'exemple, l'un d'eux raconte n'avoir pu fréquenter l'école puisqu'il n'était le «fils de personne », alors que le désarroi d'un autre est venu du fait que son père lui avait imposé un parcours scolaire. Un autre nous dit ceci :

J'allais à l'école mais j'm'organisais des fois pour rester à $m$... pour travailler à grange, parce que j'aimais ça travailler pis tout ça... Finalement, j'ai doublé ma $10^{\mathrm{e}}$ année. Là, ma sœur qui avait 11 mois de différence avec moi est venue dans la même année. Ç'a été dur pour moi, pis là, j'ai décidé de pu r'tourner à l'école. $(\mathrm{E}-271)$

\section{Parcours travail}

Au moment des entrevues, cinq participants avaient un travail à temps complet, six un travail à temps partiel, un recevait des allocations pour motif de santé, deux recevaient de l'assuranceemploi, sept recevaient de l'aide sociale (un pour invalidité) et trois étaient retraités.

Sans surprise, le parcours scolaire de plusieurs a exercé une influence considérable sur leur parcours travail. L'absence de formation a fait en sorte qu'une majorité de participants n'a pu développer de spécialité et a connu de multiples changements d'occupation. Seuls 5 sur 24 ont toujours travaillé plus ou moins dans le même domaine. Tous les autres ont exercé différents métiers et un des participants n'a pratiquement pas eu de vie professionnelle pour cause de maladie mentale. II est aussi ressorti des propos des participants que faire faillite était une expérience déterminante dans leur parcours. Cinq l'ont vécue (dont deux plus d'une fois). Un sixième s'est, quant à lui, vanté de ne « jamais avoir fait faillite ». La fréquence des références à la faillite appuie l'idée qu'elle est un risque très présent dans la vie de certains hommes et peut être mise en lien avec le rôle de pourvoyeur, symbole de masculinité. Vivre cette expérience est ainsi associé à une perte signifiante. Un autre symbole évoqué, celui de l'automobile («le char »), signifie réussite pour celui qui la possède et être propriétaire d'un modèle récent est présenté comme une manifestation d'aisance financière.

Concernant les incapacités liées au travail, quatre hommes ont mentionné avoir vécu des accidents de travail. Un homme en a subi deux et un autre plusieurs. Un cas d'épuisement professionnel a également été relevé. Dans un autre cas, le participant attribue ses problèmes de santé à son travail en imprimerie, mais n'a jamais réclamé de dédommagement. Ainsi, les risques pour la santé qui sont associés au travail sont bien présents dans le discours des participants à l'étude. 
Par ailleurs, le rapport que les hommes entretiennent avec leur travail varie d'un extrême à l'autre. Pour la majorité, le travail apparaît comme une valeur dominante. L'école est abandonnée et l'inscription sur le marché du travail suit immédiatement, souvent encouragée par les parents. Les hommes endossent dès lors le rôle de pourvoyeur. II en va toutefois autrement pour deux répondants qui se sont éloignés du modèle dominant. Ils ont choisi de s'investir dans un rôle parental de premier plan et d'assumer la majeure partie des tâches domestiques en travaillant à temps partiel alors que leurs conjointes occupaient des emplois à temps complet.

Le parcours travail des hommes résilients montre une entrée relativement précoce sur le marché du travail. Peu ont été encouragés à poursuivre des études. Sauf exception, ils ne travaillent pas dans un domaine associé à leur formation. Ce qui les distingue des non-résilients est avant tout une relative stabilité d'emploi, certains allant jusqu'à affirmer que le travail est ce qui leur permet de garder la forme et de se maintenir en santé. II arrive aussi que ces hommes doivent lutter pour se maintenir sur le marché du travail. Les propos d'un participant, dont la présence sur le marché du travail est en dents de scie, sont éloquents sur l'adversité à laquelle il doit faire face :

[...] quand j'ai tombé sur l'aide sociale, ce que j'ai trouvé le plus dur c'tait ça. Le plus insécurisant, c'est que ton maudit chèque d'aide sociale, quand t'es rendu là, tu mets autant d'énergie à pas te faire couper que d'essayer de trouver des contrats, comprends-tu? [...] j'ai assez de problèmes comme ça, là. J'ai eu des fonctionnaires qui m'appelaient pour un 35 \$ que j'avais pas déclaré y a six mois, pis là, fallait que j'trouve des papiers... Tu penses qu'y vont te couper dans les jours qui viennent, là. Pis tu vis tellement d'insécurité... [...] Moi, j'la connais la misère. Quand y parlent « eh les gens sur l'aide sociale, ça se lève à midi ». Eh oui, j'me suis levé à midi... parce que qui dort dîne! Y a des journées que j'pouvais pas me lever à $6 \mathrm{~h}$ le matin parce que j'voulais pas avoir mal au ventre à $10 \mathrm{~h}$ esti. [...] Le monde savent pas c'est quoi la misère, y parlent à travers leur chapeau. Quand quelqu'un a pas rien à manger, y est ben mieux de s'lever à midi, OK? (E-934)

Par opposition, rares sont les hommes non résilients qui ont gardé le même emploi. La majorité en a occupé plusieurs. II ressort de leurs propos, en contrepartie, qu'ils n'ont jamais souhaité ne pas travailler. Si quelques-uns reçoivent des prestations d'aide sociale, ce n'est pas à défaut de démarches, mais plutôt parce qu'ils sont disqualifiés. Le parcours d'un homme, dont nous avons déjà traité, est particulièrement troublant sur ce plan. Orphelin, il a travaillé dans des conditions s'apparentant à de l'esclavage afin de se procurer à manger et se loger. Analphabète, il n'a pu conserver un emploi. La seule période où il ne s'est pas senti exploité est celle où il a travaillé sur la ferme de son conjoint. Cependant, à la mort de ce dernier, il est retourné en mode disqualification et s'est retrouvé à l'aide sociale. 


\section{Parcours résidentiel}

Tous les répondants résident depuis au moins quatre ans dans Saint-Louis, ce qui témoigne d'un certain enracinement ${ }^{5}$. Les propos touchant le quartier ont été regroupés en trois thèmes : 1) l'attrait que constituent l'accessibilité des services (la possibilité de s'y rendre à pied) et le milieu diversifié; 2) l'aspect négatif associé à la population démunie qui y réside et l'évolution du territoire en ce sens et 3 ) le caractère Basse-Ville en opposition à la Haute-Ville, laquelle est perçue comme un milieu privilégié mais pouvant aussi être antipathique. Un participant résume ainsi :

C'est sûr que oui c't'un quartier défavorisé, mais en même temps ça dépend des familles et tout ça. C'toute mélangé pis c'est correct comme ça. C'pas la grande pauvreté non plus. T'sais on peut pas s'comparer avec l'Afrique, ou même les villes où c'est la guerre, là. Entre parenthèses, c'est des quartiers qui sont quasiment dangereux. C'est pas le cas ici. Y a des problématiques sociales, c'pas extrême pis tout ça là. Mais c'est sûr qu'y a comme des préjugés aussi... (E-867)

Avant d'arriver dans le quartier, la plupart des participants ont eu un parcours résidentiel instable. Cela ne signifie toutefois pas que le quartier n'est pas, en quelque sorte, choisi. Pour certains, il s'agit d'un retour aux sources après avoir occupé différents domiciles au gré d'unions conjugales, d'emplois, de recherches personnelles ou professionnelles, etc.

Malgré le fait que quelques hommes aient parlé de nostalgie par rapport à leur milieu d'origine et quelques autres décrit leur attachement à Saint-Louis, les propos recueillis n'ont cependant pas permis de saisir leur rapport à ce milieu de vie, ni comment celui-ci a pu contribuer à leur parcours. Cependant, il en ressort une représentation des lieux habités comme découlant de choix circonstanciels plutôt que d'un choix de milieu où habiter. En revanche, le rapport au domicile a souvent été décrit, notamment lorsqu'il y a eu propriété de maison ou accès à un logement décent. L'expérience de propriété, vécue par certains, a toutefois pris fin, pour des raisons financières (faillite) ou conjugales (ruptures).

La question de l'accès à un logement décent a été soulevée principalement par les participants dont la situation financière se révèle précaire. Leurs récits confirment l'importance de cet accès lors des périodes de fragilisation. Un des participants a, par exemple, raconté comment l'accès à un logement social avait déterminé sa trajectoire et celle de sa famille, leur offrant de la sécurité alors que ses enfants fréquentaient l'école. Pour cet homme, n'eût été de cette option, la situation se serait dégradée. Deux autres participants, fragilisés par des troubles de santé mentale et bénéficiant d'un tel logement grâce à leur mère, plaident également pour un tel soutien. Selon les propos recueillis, l'accès à un logement subventionné donne également accès à un statut social.

5. Un des répondants avait quitté Saint-Louis. Nous avons toutefois conservé son entrevue car il y avait habité pendant plusieurs années et résidait à proximité du quartier. 
Dans les récits, l'accès au logement décent est évoqué comme un luxe, une chance ou un privilège. Cela s'avère particulièrement fondé pour les chambreurs pour qui un tel logement semble hors de portée. Ainsi, nous avons noté que le parcours des non-résilients se caractérise par le fait qu'ils vivent dans Saint-Louis en raison, notamment, de la présence de logements sociaux, de logements supervisés ou de maisons de chambres.

\section{Parcours santé et ressources}

Bien que certains des hommes rencontrés aient vécu des traumatismes, des interventions d'ordre médical ou souffrent de maladies chroniques, leur discours montre qu'ils accordent plus d'importance à la santé mentale qu'à la santé physique. Les problèmes de santé mentale ressortent comme le lieu d'expériences difficiles pour plusieurs. Certains ont été confrontés à cette réalité à travers des personnes de leur entourage, ce qui a marqué leur trajectoire. Ainsi, leurs propres difficultés sur le plan de la santé mentale doivent être considérées en rapport avec des relations difficiles, particulièrement au cours de l'enfance. Pour d'autres, ce sont des événements de la vie qui les ont fragilisés.

Des problèmes de dépendance (alcool ou drogues) sont évoqués dans 10 récits (épisodes circonscrits à la jeunesse pour deux participants). Parmi les consommateurs, trois avaient été exposés à l'alcoolisme de leur père et un à celui de ses deux parents. Le fils de l'un de ces quatre répondants avait aussi consommé à son tour. Ces quatre répondants ont aussi été victimes de violence pendant leur enfance. Enfin, deux hommes ne consommant pas du tout ont eu, pour leur part, une conjointe alcoolique.

Huit participants ont mentionné avoir vécu des épisodes de dépression. Quatre ont été traités en psychiatrie (dépression ou autre trouble). Pour sa part, un participant a affirmé que c'est en travaillant à temps partiel qu'il protège sa santé mentale jugée fragile. Un autre dit plutôt avoir choisi l'automédication par peur d'être stigmatisé par son médecin.

Le suicide est aussi évoqué dans les propos des hommes. Deux participants ont raconté avoir tenté de s'enlever la vie. Un participant confie avoir perdu son unique frère par suicide et un autre que sa mère a fait une telle tentative. Enfin, deux hommes ont traversé un deuil difficile, ayant perdu un collègue par suicide.

On est allés chez lui, on a cogné, cogné... On a cogné chez le voisin... Y avait le fils du voisin qui était là. On a dit: "On voudrait rentrer chez $X$ ». Le gars cherchait les clés, [...] pis finalement on a regardé à la fenêtre du sous-sol, y était pendu là. [...] Pour moi ç'a été un $\mathrm{CHOC}$, un $\mathrm{CHOC}$ mon gars là... [...] En tout cas... Chu retourné au travail pis... Là, moi j'ai voulu instaurer les sentinelles en milieu de travail... [...] J'ai présenté mon projet. Ç'a été refusé naturellement. [...] pis j'ai eu besoin d'un psychologue aussi là quand même, pour stress posttraumatique hein... J'ai subi une dépression... J'ai voulu le rejoindre aussi... (E-72) 
Concernant la violence, en sus des cas mentionnés plus haut, d'autres répondants ont parlé de violence dans leur enfance : l'orphelin a subi plusieurs viols; un participant avait un père violent; un autre avait été maltraité par ses frères et deux avaient été victimes de violence à l'école. Enfin, un participant a raconté la violence psychologique subie par sa mère de la part de son père.

Du côté de la santé physique, les récits révèlent que plusieurs hommes ont des limitations physiques ou consomment des médicaments signalant des problèmes cardiaques, du diabète, des interventions chirurgicales, etc. Mais l'importance accordée à la recherche d'un équilibre mental a pour effet de diminuer celle accordée aux problèmes de santé physique. Ainsi, les propos sur les ressources utilisées en santé physique sont moins détaillés et moins critiques (positivement ou négativement) que ceux touchant à la santé mentale. La santé physique reste tout de même un déterminant du parcours de certains (bec-de-lièvre marquant une personnalité, perte d'un membre suite à un accident, accidents de travail, allergies développées au travail, obésité).

Lorsque nécessaire, plusieurs ont trouvé les ressources pour appuyer leur réseau personnel. C'est le cas de ce participant éprouvé par le suicide d'un collègue. Lorsque ce soutien est donné par la famille, on remarque l'activation de liens tissés dans l'enfance. D'autres ont trouvé du soutien auprès d'un voisin, une voisine ou un propriétaire compréhensif et aidant. Ces appuis ont su se révéler déterminants à un moment ou un autre.

Les informations transmises quant au rapport aux ressources professionnelles ou communautaires (sociales et de santé) se sont révélées très riches. Les participants racontent comment ils ont été soutenus à certains moments de leur vie. Le soutien y est généralement décrit en termes positifs et provient de différents professionnels et organismes (médecin, psychologue, organisme communautaire). Ces derniers ont donné un coup de main en relation à différents besoins (alimentaires, psychologiques, logement, etc.).

Fait que, pour m'en sortir [drogue, délinquance], j'ai décidé de d'mander rien à personne. J'ai couché à la maison de Lauberivière. J'allais manger dans les places qui donnaient à manger aux démunis. J'attendais ma place en thérapie. Quand j'suis rentré en thérapie, ben... j'ai arrêté de consommer. [...] Si un jour je deviendrais riche, j'gagnerais, euh, des millions là, tu peux être sûr, tu peux être sûr que la Maison Lauberivière pis la Maison Revivre ça serait deux organismes que j'aurais très à cœur (silence). Pour moi, ç'a été une expérience de vie... Je me suis surpris, à un moment donné, de m'plaindre. Pis quand j'me suis comparé à des gens qui sont là, je me suis trouvé chanceux. Très, très, très chanceux. (E-196)

Cela dit, certains ont évoqué des ressources qui n'avaient pu leur offrir le soutien dont ils auraient eu besoin. II ressort que certains appels à l'aide n'ont pas trouvé de réponse. Le cas échéant, leurs critiques sont ciblées et portent sur une situation ou une ressource précise. Quoi qu'il en soit, une chose reste cependant certaine : le besoin de ressources est souligné dans une grande majorité de récits, voire parfois à plusieurs reprises. 
Dans ce parcours, la distinction entre les hommes résilients et ceux qui ne le sont pas tient surtout au fait que, bien que les résilients ne soient pas à l'abri de la dépression ou d'idées suicidaires, ils font mentir le cliché voulant que « les hommes » ne consultent pas. Ces hommes vont chercher de l'aide, s'inscrivent parfois en thérapie (consommation d'alcool ou état dépressif) et affirment prendre leur médication telle que prescrite pour surmonter leurs problèmes.

Si, à l'occasion, ils demandent de l'aide pour surmonter leurs problèmes, les non-résilients se caractérisent de façon générale par une non-utilisation des ressources ou un manque de confiance en ces dernières. Cela s'applique à tous les services étatiques. Leur rencontre avec les intervenants est rarement heureuse: s'il leur arrive d'avoir des expériences positives (écoute, aide et soutien), il n'est pas rare de voir le lien se rompre, surtout dans les cas de problèmes de santé mentale ou de changements de territoire administratif.

\section{Parcours liens sociaux}

De manière générale, les hommes qui ont participé à notre étude avaient relativement peu d'amis. Aucun n'a fait le récit d'une amitié profonde et actuelle, les liens décrits en étaient davantage de camaraderie. Ainsi, sur le plan social, ces hommes paraissent plutôt seuls. Bien qu'ils aient peu d'amis, les résilients en ont tous au moins un. Certains relatent avoir vécu une amitié profonde dans leur jeunesse, mais aucune actuelle, ces liens étant aujourd'hui coupés. Les hommes résilients ne semblent pas se confier beaucoup, sauf lorsqu'ils sont en couple, situation où la conjointe apparaît comme la seule confidente.

Pour leur part, les non-résilients sont des hommes seuls avec peu ou pas d'amis. II peut arriver qu'ils se confient à une amie, à leur conjointe ou à une ex-conjointe, mais de façon sporadique. Certains ont aussi, par le passé, développé des liens à l'intérieur d'un gang ou d'une secte religieuse.

\section{DiscusSION DES RÉSULTATS}

\section{Famille, enfance et figures d'attachement}

Le premier environnement où évolue une personne est sa famille. Ce lieu de filiation influe sur l'identité. La richesse ou la pauvreté du capital social et culturel de ce milieu influencera l'enfant qui y grandit, l'adéquation ou l'inadéquation de la bulle affective qui s'y construit le détruisant ou lui fournissant le matériel nécessaire pour tricoter sa résilience (Cyrulnik, 1999).

Les récits révèlent qu'une enfance malmenée peut avoir des effets dévastateurs. En ce sens, plusieurs études effectuées auprès d'enfants de parents alcooliques (notamment Tousignant, 1999) illustrent l'importance d'une figure substitut pour donner un sens à la vie. De même, les enfants victimes de violence ou d'agressions émanant de personnes proches risquent davantage de développer de la détresse psychologique (Martinez et Richters, 1993; Jernkins et Bell, 1995). L'absence des pères et des mères, tout comme leur omniprésence, marquent tout autant le parcours de vie de leurs enfants. Comme le mentionne Tousignant, « l'enfant comblé peut être aussi vulnérable que l'enfant à haut risque psychosocial qui, lui, a 
souvent su développer des mécanismes de résistance qui font qu'il ne s'écroulera pas au premier sacrifice exigé » (1999: 133).

Nos travaux permettent aussi d'identifier des éléments pouvant modifier les effets défavorables de moments de vie difficiles. Comme Long et Vaillant (1989), nous avons vu que la présence d'un adulte substitut (tante ou oncle) ou encore un bon mariage sont des indicateurs de résilience. De plus, la présence d'une relation étroite avec au moins une figure parentale constituerait le prédicteur le plus solide concernant l'avènement d'aptitudes de résilience (Luthar, 2006; Luthar et Zelazo, 1994; Masten, 2001; Rutter, 2000). Le chercheur Égide Royer (2010 : 45) souligne que « la théorie de l'Attachement postule que notre capacité d'entrer en relation, de développer des liens avec d'autres êtres humains, est tributaire du type de relation que nous avons eu avec nos figures d'attachement primaire, la plupart du temps nos parents ». De telles données portent donc à croire que la résilience n'est pas qu'un attribut personnel, mais qu'il s'agit plutôt d'une capacité qui se construit dans l'interaction d'une personne avec son environnement social.

\section{École, violence et masculinités}

Le parcours scolaire apparaît comme très significatif dans les trajectoires de vie des hommes interrogés. Devant l'ensemble des difficultés rencontrées, les récits, tant des résilients que des non-résilients, font écho au phénomène du décrochage scolaire qui, nous le savons, affecte de manière exacerbée les garçons (Royer, 2010). Cet aspect du vécu masculin est préoccupant compte tenu des liens entre le niveau de scolarité, le statut social et économique et l'état de santé, bref, le parcours de vie en général (Frohlich, Corin et Potvin, 2008; Quesnel-Vallée, 2008; Ruxton, 2002). Or, nos données font penser que l'élément central de résilience par rapport à l'école ne serait pas tant la qualité des résultats scolaires que la capacité des hommes à intégrer leur parcours scolaire dans un plan de carrière, carrière souvent orientée vers un rôle de pourvoyeur. Dans ce contexte, il y a lieu de s'alarmer de ce que deviendra le profil de santé des hommes si les taux de décrochage scolaire des garçons continuent de s'accentuer et ce, même si le rôle de pourvoyeur n'est plus aussi caractéristique de l'homme qui réussit. II nous apparaît important de militer en faveur d'actions dirigées vers les milieux d'éducation afin de soutenir les jeunes garçons dans la poursuite de leurs études, minimalement jusqu'à l'obtention d'un diplôme.

Les données recueillies mettent aussi en évidence la violence et l'humiliation vécues dans les parcours scolaires. Dans la cour d'école, les jeunes garçons sont forcés d'endosser les rapports de force permanents liés à une certaine idéologie masculine sous peine de devenir des victimes désignées (Ayral, 2011; Dorais, 2000; Welzer-Lang, 1994) et de s'attirer les assauts de leurs camarades. Le sociologue Pierre Merle estime que cette violence et cette humiliation vécues par les garçons est également présente dans les salles de classe (Merle, 2005). Bien que nous ne sachions comment intervenir pour soutenir les garçons qui s'y sentent mal à l'aise, nous pouvons toutefois affirmer que l'école constitue un lieu où il est important d'intervenir pour que ces derniers puissent s'y épanouir en toute sécurité. Cela sera bénéfique évidemment pour les garçons eux-mêmes, mais aussi pour le bien-être de leurs enfants, des partenaires qui partageront leur vie, de leur famille et de la société tout entière. 


\section{Le travail}

Cyrulnik (1999) estime que le choix d'un métier (avec le choix du conjoint) est l'un des choix les plus névralgiques de notre existence. Or, le parcours travail des hommes rencontrés est lié au parcours familial, en ce sens que, sauf exception, les fils ont, tout comme leurs pères, entièrement investi leur travail. Le rôle de pourvoyeur est demeuré central pour plusieurs. Ce constat fait écho à ce que d'autres ont déjà mis en lumière en ce qui concerne la génération des hommes rencontrés. En 1982, O'Neil affirmait que le travail et la carrière d'un homme étaient perçus, par les hommes eux-mêmes et par la société dans son ensemble, comme la mesure « étalon » de sa masculinité (succès, compétence, accomplissement). L'importance que les hommes accordaient au travail et au revenu qui l'accompagne pour se garantir possessions et sécurité constituait ce qu'O'Neil (1982) appelait le « syndrome du pourvoyeur ». En milieu de pauvreté à plus forte raison, l'homme pourvoyeur était le modèle de l'accomplissement de soi masculin. Les chercheurs australiens Paris et Vickers (2010) estiment que, encore aujourd'hui, dans plusieurs cultures occidentales, l'homme a toujours un rôle de pourvoyeur et que son entourage s'attend à ce qu'il subvienne aux besoins de sa famille. Toutefois, avec le changement des mentalités dans les sociétés contemporaines, les jeunes tendent à délaisser ce modèle. II n'en demeure pas moins que le travail demeure un élément clé de la définition de la masculinité et construit l'identité des répondants de cette étude. La perte de leur emploi, le cas échéant, eut un impact dévastateur sur leur estime personnelle, leur faisant ressentir qu'ils avaient failli à leurs responsabilités. C'est sans surprise que Crowley (1998) constate le lien, chez de nombreux hommes, entre la dépression et les conflits conjugaux, d'une part, et d'autre part le sentiment d'incompétence quant à la qualité de pourvoyeur. Après avoir insisté durant des générations sur l'importance du rôle de pourvoyeur du père, il apparaît un peu utopique de penser que cette pression sociale va s'évaporer du jour au lendemain (Crowley, 1998). À preuve, dans notre étude, même les hommes disqualifiés accordaient une grande importance à cette valeur travail. D'ailleurs, cette pression contribuerait au fait que beaucoup d'hommes consacrent plus d'énergie à leur travail qu'à leur famille (Tremblay, 2005).

II ressort également de notre analyse que, chez les hommes considérés comme résilients, certains se trouvent capables de réaliser une profonde et salutaire rupture avec ce rôle de pourvoyeur si central dans la masculinité traditionnelle. Selon Allard, Bourret et Tremblay (2004), ce lâcher-prise envers la valeur travail permettrait aux pères séparés vivant en milieu de pauvreté de développer une paternité ouverte et engagée, phénomène qui nous semble confirmé par nos propres données. Un tel constat mène à penser que l'on devrait encourager les hommes à diversifier leurs investissements dans différentes sphères de leur vie.

\section{Le logement}

Plusieurs recherches indiquent qu'un logement stable constitue un déterminant social important qui a un impact sur la santé (Laurence, 2010) et que l'accès à un logement permet d'alimenter et de soutenir un processus d'amélioration des conditions sociales et psychologiques (Halpern, 1995). L'ensemble des témoignages recueillis vont dans ce sens. L'octroi d'un logement, bien que dans un quartier moins nanti, a été un tremplin pour plusieurs. Fait particulier, la distinction entre les hommes considérés comme résilients et les non-résilients semble tenir surtout à ce qui les amène dans Saint-Louis: circonstances en rapport avec l'école ou le travail pour les 
résilients, présence de logements sociaux et de maisons de chambres pour les moins résilients. Dans ces cas-ci, « la stabilité d'un logis en tant que réel "chez-soi" constitue un port d'ancrage déterminant pour les personnes psychologiquement et socialement instables » (Morin, Robert et Dorvil 2001 : 96), mais de façon générale, elle évite également une fragilisation des situations vécues par les hommes plus stables. Qu'il appartienne au groupe des résilients ou pas, chacun des hommes semble savoir profiter des ressources du quartier puisque « [l]'accès à un logement adéquat et abordable établit les circonstances et/ou les opportunités d'accessibilité à d'autres supports réseau formels et informels » (Carter et Polevychok, 2004 : 3). Un tel constat pointe donc vers la nécessité de dépasser le paradigme de la «personne à risque » pour prendre en considération les « contextes à risque », la précarité du logement étant un facteur de vulnérabilité important pour tous.

\section{La santé physique et mentale des hommes}

Dans la première monographie québécoise portant sur la santé des hommes, il est mentionné qu'à « tous les âges, les hommes déclarent moins de détresse psychologique que les femmes et que les hommes sont proportionnellement plus nombreux à se percevoir comme étant en meilleure santé mentale que les femmes »(Québec, 2005: 139). Les hommes consultent également moins les services de santé et sociaux que les femmes. Nos données ne permettent pas de comparaison avec une population féminine, mais elles révèlent une fréquence importante de détresse élevée et de problèmes de santé mentale chez nos participants. Nous avons aussi vu que, parmi les résilients, certains étaient allés chercher de l'aide à des moments opportuns pour surmonter leurs difficultés alors que, pour les non-résilients, la relation avec les services d'aide était difficile. Or, il est connu que, de manière générale, les hommes sont réticents à y faire appel, attendant souvent jusqu'à la crise, ce système étant jugé peu adapté à leur réalité (Dulac, 2001). II semble donc nécessaire de mieux comprendre quels sont les obstacles rencontrés par les hommes dans leurs rapports avec le système de soins afin d'agir sur les facteurs ainsi mis au jour. À partir de nos résultats, nous estimons qu'il s'agit de favoriser, chez les hommes résilients, un recours plus rapide à l'aide et, chez les non-résilients, de stimuler la persévérance dans leurs démarches pour ainsi augmenter leurs chances d'améliorer leur bien-être.

Nos résultats ont aussi illustré la place significative occupée par la santé mentale dans les parcours de vie des hommes. Notons ici que le suicide est une épreuve que certains ont rencontrée, soit pour avoir eu des idées suicidaires, pour avoir fait des tentatives ou pour avoir vécu un deuil par suicide. Bien que dans les pays industrialisés, comme au Québec, trois à quatre fois plus d'hommes que de femmes décèdent par suicide (Robertson, 2007; Ruxton, 2002; St-Laurent et Bouchard, 2004), ce sont surtout les différents facteurs de vulnérabilité présents au sein des parcours de nos participants qui retiennent notre attention. D'une part, plusieurs ont eu à composer avec la dépression, facteur intimement lié au suicide. D'autre part, la grande importance qu'ils accordent à la valeur travail et au rôle de pourvoyeur pourrait constituer un facteur de détresse grave chez ceux qui n'arrivent pas à remplir ce rôle. Ces derniers, ne pouvant répondre à cette attente sociale liée à la masculinité, éprouveraient une forte honte résultant d'une intériorisation de leur échec (De Gaulejac, 1996). Notons à cet égard 
qu'il a été démontré par Houle (2005) que l'adhésion rigide à des représentations traditionnelles de la masculinité, dont le rôle de pourvoyeur fait partie, augmentait les risques de suicide.

\section{Amitié et liens sociaux}

Une grande solitude ressort des récits que nous ont livrés les hommes qui ont participé à notre étude. Ils ont, pour la plupart, peu ou pas d'ami à qui se confier ou sur qui compter. Nous avons toutefois constaté que les résilients pouvaient, en général, compter sur au moins un lien d'amitié, lien souvent établi avec la conjointe. Ces données font écho à ce qui est depuis longtemps connu quant aux déficits de l'intimité masculine. «La littérature des années 1970 laisse entendre que l'amitié masculine est exceptionnelle et que l'intimité est une expérience rare parmi les hommes américains. Certes, ces derniers forment un large réseau social, mais ils n'ont pas d'amis intimes comme à l'adolescence » (Levinson, 1978: 335, cité dans Dulac, 2003 :13). Cette solitude a, selon nous, quelque chose d'alarmant puisque, dans sa forme la plus intense, elle contribue aux passages à l'acte suicidaire. Si les liens sociaux sont indispensables à la résilience, cet isolement des hommes, qui semble peu évoluer à travers le temps, ne peut qu'être déploré. Les hommes résilients sont ceux qui entretiennent un minimum de liens dans leur entourage et savent aller en bâtir d'autres avec des services d'aide lorsque nécessaire. C'est en ce sens que Nantel et Gascon (2002) proposent, pour mieux aider les hommes, de favoriser le renforcement des liens que ces derniers entretiennent avec les intervenants, le réseau d'aide formel, les réseaux sociaux et la société dans son ensemble. Cependant, considérant que le pouvoir, dans la société, est réparti de façon inéquitable entre les hommes eux-mêmes, il paraît clair que sans une volonté publique d'aider les hommes en instaurant des mesures concrètes pour faciliter ces différents liens, il est difficile de présager un avenir meilleur.

\section{Conclusion}

Notre étude auprès d'hommes de 45 à 64 ans d'un quartier urbain de la région de Québec confirme la pertinence d'approfondir les connaissances sur la santé des hommes. En effet, la situation de défavorisation dans laquelle ils se retrouvent et les problèmes de santé éprouvés semblent être les résultats d'une construction sociale dans laquelle leurs représentations de la masculinité jouent un rôle déterminant. Véritable entrave à l'expression d'émotions, au développement de liens sociaux, les rôles masculins traditionnels sont souvent source de colère ou d'insatisfactions face à une vie qui ne permet pas de répondre à ses aspirations ni aux attentes des autres. Les expériences vécues dans la petite enfance ont été déterminantes pour les hommes rencontrés, tout comme pour l'autre groupe sur lequel s'est penchée cette recherche (les responsables de familles monoparentales). Toutefois, dans le cas des hommes, le parcours scolaire, compte tenu des attentes liées au rôle de pourvoyeur, ressort davantage comme un facteur important dans leurs trajectoires de vie. Pour les plus souffrants, l'école apparaît comme un lieu inhospitalier où ils n'ont pu trouver l'écoute et l'encouragement qui leur auraient permis d'y acquérir la maturité et les outils nécessaires pour assurer leur avenir. Ensuite, les lacunes affectives ressenties à différents moments de leur vie, alors que la vie affective des hommes n'est pas valorisée, semblent avoir blessé particulièrement ceux qui s'en sortent le moins bien, pour qui la quiétude mentale paraît plus difficilement accessible. À 
l'inverse, les récits d'autres hommes rencontrés montrent que l'affection qui leur a été manifestée dans l'enfance ou dans leur vie amoureuse les a aidés à se faire une place au soleil. Finalement, l'histoire de deux hommes rencontrés, qui sont en quelque sorte des contremodèles quant à la masculinité traditionnelle, illustre bien qu'une remise en question du rôle imparti aux hommes peut aider à se réaliser de façon globale. La contribution de cette étude consiste donc en une ouverture sur ce champ de recherche à développer qu'est la santé des hommes. D'autres travaux seront nécessaires afin de repérer des façons de faire qui permettront aux garçons et aux hommes de briser le carcan du rôle masculin traditionnel lorsqu'il nuit à leur épanouissement et au développement de relations saines avec les femmes et avec leurs enfants.

L'étude apporte aussi une contribution sur le plan méthodologique. D'une part, l'approche utilisée, soit celle des parcours de vie, s'est confirmée comme porteuse de sens. Les instruments que nous nous sommes donnés, en construisant une grille chronologique décomposée en parcours thématiques, ont grandement facilité l'exploitation du matériel recueilli. Nous avons pu ainsi cerner les lieux et expériences déterminants dans les cheminements des hommes rencontrés. Par conséquent, il peut être envisageable que l'utilisation à plus grande échelle de cette approche et de nos outils faciliterait le repérage de lieux d'intervention pouvant se révéler efficaces. D'autre part, l'analyse des trajectoires individuelles dans le but de les distinguer selon la présence ou non de résilience constitue une innovation méthodologique fort intéressante. En procédant ainsi, il nous a été possible de mieux comprendre pourquoi et quand certaines expériences contribuent à la capacité qu'ont les hommes de s'en sortir une fois adultes dans différentes sphères de vie, ou inhibent cette capacité.

En terminant, nous espérons que cette étude n'est que le premier pas qui précède d'autres travaux. Les intervenantes et intervenants vers qui se tournent ou ne se tournent pas les hommes en détresse ont besoin d'informations et d'analyses sur les expériences de ceux-ci. Sur ce point, nous sommes convaincus que la recherche est nécessaire, voire même urgente. De plus, elle pourrait soutenir une remise en cause de la définition encore dominante et délétère de la masculinité pour lui en substituer une autre plus salutaire, c'est-à-dire une approche qui tirerait profit des forces présentes dans les masculinités.

Bernard Roy, Ph.D

Professeur agrégé, Faculté des sciences infirmières, Université Laval

Maria De Koninck, sociologue de la santé Professeure retraitée, Département de médecine sociale et préventive, Université Laval

Michelle Clément, chercheure CSSS de la Vieille-Capitale, centre affilié universitaire

Éric Couto, M.S.S., T.S. Étudiant doctorant en service social, Équipe Masculinités et Société, Université Laval 


\section{BIBLIOGRAPHIE}

Allard, F. L., A. Bourret et G. Tremblay (2004). Rester engagé envers son enfant après la rupture du couple : point de vue de pères vivant en contexte de pauvreté, Québec, Direction régionale de santé publique de la Capitale nationale.

Ayral, S. (2011). La fabrique des garçons. Sanctions et genre au collège, Paris, Presses universitaires de France.

Bernard, P., C. Beach, P. Bélanger, J. Charbonneau, C. Le Bourdais, D. Livingstone et al. (2006). Capturing the lifecourse. The contribution of a Panel Study of Lifecourse Dynamics (PSLD) to public policy analysis in Canada, A report on the opportunity and feasibility of the PSLD, submitted to Social Development Canada.

Carter, T. et C. Polevychok (2004). Housing is good social policy. Ottawa: Canadian Policy Research Networks.

Crowley, M. Sue (1998). «Men's Self-Perceived Adequacy as the Family Breadwinner: Implications for Their Psychological, Marital, and Work-Family Well-Being », Journal of Family and Economic Issues, vol. 19, $\mathrm{n}^{\circ}$ 1, p. 7-23.

Cyrulnik, B. (1999). Un merveilleux malheur, Paris, Odile Jacob.

De Gaulejac, Vincent (1996). Les sources de la honte, Paris, Desclée de Brouwer.

De Koninck, M. (2008). « Un regard multidisciplinaire sur la construction des inégalités sociales de santé », dans K. Frohlich, M. De Koninck, A. Demers et P. Bernard (dir.), Les inégalités sociales de santé au Québec, Montréal, Les Presses de l'Université de Montréal, p. 57-84.

Dulac, G. (2001). Aider les hommes aussi, Montréal, VLB.

Dulac, G. (2003), Masculinité et intimité. Sociologie et sociétés, vol. 35, n² 2, p. 9-34.

Dorais, M. (2000). Mort ou fif, Montréal, VLB.

Dupéré, S. et M.-J. Disant (2005). Réflexion sur les ancrages théoriques du concept de pauvreté, Québec, Département de médecine sociale et préventive, Université Laval.

Équipe de recherche sur les inégalités sociales de santé (2006a). Inégalités de santé et milieux de vie: déterminants sociaux en cause et leurs interactions. Monographie de Limoilou, Québec, Département de médecine sociale et préventive de I'Université Laval, INSPQ, CSSS de la Vieille-Capitale.

Équipe de recherche sur les inégalités sociales de santé (2006b). Inégalités de santé et milieux de vie : déterminants sociaux en cause et leurs interactions. Monographie de Charlesbourg, Québec, Département de médecine sociale et préventive de I'Université Laval, INSPQ, CSSS de la Vieille-Capitale.

Équipe de recherche sur les inégalités sociales de santé (2006c). Inégalités de santé et milieux de vie: déterminants sociaux en cause et leurs interactions. Monographie de Portneuf, 
Québec, Département de médecine sociale et préventive de I'Université Laval, INSPQ, CSSS de la Vieille-Capitale.

Équipe de recherche sur les inégalités sociales de santé (2006d). Trois territoires, trois réalités. Éléments d'analyse comparative sur la construction des inégalités sociales de santé, Québec, Département de médecine sociale et préventive de l'Université Laval, INSPQ, CSSS de la Vieille-Capitale.

Frohlich, K, E. Corin et L. Potvin (2008). «La relation entre contexte et maladie: une proposition théorique », dans Katherine Frohlich, Maria De Koninck, Andrée Demers et Paul Bernard (dir.), Les inégalités sociales de santé au Québec, Montréal, Les Presses de l'Université de Montréal, p. 141-185.

Halpern, D. (1995). Mental health and the built environment: More than bricks and mortar? London, Taylor \& Francis.

Houle, J. (2005). La demande d'aide, le soutien social et le rôle masculin chez les hommes qui ont fait une tentative de suicide. Thèse de doctorat, Département de psychologie, Université du Québec à Montréal, Montréal.

Jernkins, E. et C. Bell (1995). « Violence exposure, psychological distress, and risk behaviors among inner-city high school students », dans S. Friedman (dir.), Anxiety disorders in African-Americans, New York, Springer, p. 76-88.

Krieger, N. (2001). «A glossary for social epidemiology », Journal of Epidemiology and Community Health, vol. 55, $\mathrm{n}^{\circ}$ 10, p. 693-700.

Kuh, D., Y. Ben-Shlomo, J. Lynch, J. Hallqvist et C. Power (2003). « Life course epidemiology », Journal of Epidemiology and Community Health, vol. 57, $\mathrm{n}^{\circ} 10, \mathrm{p} .778-783$.

Laurence, R.J. (2010). « Housing and health promotion: moving forward, International Journal of Public Health, vol. 55, $\mathrm{n}^{\circ}$ 3, p. 145-146.

Lebel, A., R. Pampalon et P. Villeneuve (2007). «An integrated approach for defining neighbourhood units in the context of a study on health inequalities in the Quebec City region », International Journal of Health Geographics, vol. 6, nº 27.

Lefebvre, C. (2004). Un portrait de la santé des hommes québécois de 30 à 64 ans, Québec, Institut national de santé publique du Québec.

Long, J. et G. Vaillant (1989). « Escape from the underclass », dans T. Dugan et R. Coles (dir.), The Child in Our Times. Studies in the Development of Resiliency, New York, Guilford Press, p. 200-213.

Luthar, S. (2006). «Resilience in development: A synthesis of research across five decades », dans D. Cicchetti et D.J. Cohen (dir.), Developmental Psychopathology: Risk, disorder, and adaptation, New York, Wiley, p. 740-795.

Luthar, S. et L. Zelazo (1994). « Research on resilience: An integrative review », dans S. Luthar (dir.), Resilience and vulnerability: Adaptation in the context of childhood adversities, New York, Cambridge University Press, p. 510-549. 
Martinez, P. et J. Richters (1993). «The NIMH community violence project: II. Children's distress symptoms associated with violence exposure », Psychiatry, vol. 56, p. 22-35.

Masten, A. (1994). «Resilience in individual development: Successful adaptation despite risk and adversity », dans M. Wang et E. Gordon (dir.), Educational resilience in inner-city America: Challenges and prospects, Hillsdale, Erlbaum, p. 3-25.

Masten, A. (2001). «Ordinary magic: Resilience process in development », American Psychologist, vol. 56, $\mathrm{n}^{\circ}$ 3, p. 227-238.

Masten, A., K. Best et N. Garmezy (1990). « Resilience and development: Contribution from the study of children who overcome adversity », Development and Psychopathology, vol. 2, $\mathrm{n}^{\circ} 4$, p. 425-444.

Morin, P., D. Robert et H. Dorvil (2001). « Le logement comme facteur d'intégration sociale pour les personnes classées malades mentales et les personnes classées déficientes intellectuelles », Nouvelles pratiques sociales, vol. 14, n² 2, p. 88-105.

Nantel, Y.C. et É. Gascon (2002). «Les difficultés masculines et l'intervention sociale : Une question de liens? » Intervention, $\mathrm{n}^{\circ} 116$, p. 103-111.

O'Neil, J.M. (1982). «Gender-role conflict and strain in men's lives. Implications for psychiatrists, psychologists, and other human service providers », dans K. Solomon et N. Levy (Eds.), Men in transition - Theory and therapy, New York, Plenum Press, p. 5-44.

Merle, P. (2005). L'élève humilié : L'école, un espace de non-droit?, Paris, Presses universitaires de France.

Pampalon, R. et G. Raymond (2000). « Un indice de défavorisation pour la planification de la santé et du bien-être au Québec », Maladies chroniques au Canada, vol. 21, n ${ }^{\circ}$, p. 113122.

Parris, M.A. et H.V. Margaret (2010). « "Look at Him... He's Failing”: Male Executives' Experiences of Redundancy », Employee Responsibilities and Rights Journal, vol. 22, $\mathrm{n}^{\circ} 4$, p. 345-357.

[http://www.springerlink.com/index/10.1007/s10672-010-9156-9], (Consulté le 19 octobre 2011).

Quesnel-Vallée, A. (2008). «L'approche des parcours de vie », dans K. Frohlich, M. De Koninck, A. Demers et P. Bernard (dir.), Les inégalités sociales de santé au Québec, Montréal, Les Presses de l'Université de Montréal, p. 221-242.

Reading, J. (2009). Les déterminants sociaux de la santé chez les Autochtones: Approche fondée sur le parcours de vie, Ottawa, Sous-comité sénatorial sur la santé de la population.

Robertson, S. (2007). Understanding men and health. Masculinities, identity and well-being, Berkshire, Open University Press.

Roy, S. (2008). « De l'exclusion à la vulnérabilité. Continuité et rupture », dans V. Châtel et S. Roy (dir.), Penser la vulnérabilité. Visages de la fragilisation du social, Québec, Presses de l'Université du Québec, p. 13-34. 
Royer, É. (2010). Leçons d'éléphants. Pour la réussite des garçons à l'école, Québec, École et comportement.

Rutter, M. (1987). « Parental mental disorder as a psychiatric risk factor », dans R. Halers et A. Frances (dir.), American Psychiatric Association annual review, Washington, American Psychiatric Association, p. 647-663.

Rutter, M. (1990). «Psychosocial resilience and protective mechanisms », dans J. Rolf, A. Masten, D.N. Cicchetti et S. Weintraub (dir.), Risk and protective factors in the development of psychopathology, New York, Cambridge, p. 181-214.

Rutter, M. (2000). « Resilience reconsidered: Conceptual considerations, empirical findings, and policy implications », dans J. Shonkoff et S. Meisels (dir.), Handbook of early childhood intervention, $2^{\mathrm{e}}$ éd., New York, Cambridge University Press, p. 651-681.

Ruxton, S. (2002). Men, masculinities and poverty in the UK, Oxford, Oxfam.

St-Laurent, D. et C. Bouchard (2004). L'épidémiologie du suicide au Québec : que savons-nous de la situation récente?, Québec, Institut national de santé publique du Québec.

[http://www.inspq.qc.ca/pdf/publications/283-FeuilletEpidemioSuicide.pdf] (Consulté le 19 octobre 2011).

Tousignant, M. (1999). « De l'adversité à la résilience: la part du milieu, la part de l'enfant ». PRISME, vol. 29, p. 122-135.

Tremblay, G. (2005). La santé des hommes au Québec, Québec, Ministère de la santé et des services sociaux, Institut de la statistique du Québec.

Welzer-Lang, D. (1994). «L'homophobie, la face cachée du masculin », dans D. Welzer-Lang, P.-J. Dutey et M. Dorais (dir.), La peur de l'autre en soi, du sexisme à l'homophobie, Montréal, VLB, p. 13-92. 